\title{
Application of a curated genome-scale metabolic model of CHO DG44 to an industrial fed-batch process
}

\author{
Calmels, Cyrielle; McCann, Andréa; Malphettes, Laetitia; Andersen, Mikael Rørdam
}

Published in:

Metabolic Engineering

Link to article, DOI:

10.1016/j.ymben.2018.09.009

Publication date:

2018

Document Version

Peer reviewed version

Link back to DTU Orbit

Citation (APA):

Calmels, C., McCann, A., Malphettes, L., \& Andersen, M. R. (2018). Application of a curated genome-scale metabolic model of CHO DG44 to an industrial fed-batch process. Metabolic Engineering, 51, 9-19. https://doi.org/10.1016/j.ymben.2018.09.009

\section{General rights}

Copyright and moral rights for the publications made accessible in the public portal are retained by the authors and/or other copyright owners and it is a condition of accessing publications that users recognise and abide by the legal requirements associated with these rights.

- Users may download and print one copy of any publication from the public portal for the purpose of private study or research.

- You may not further distribute the material or use it for any profit-making activity or commercial gain

- You may freely distribute the URL identifying the publication in the public portal 


\section{Author's Accepted Manuscript}

Application of a curated genome-scale metabolic model of CHO DG44 to an industrial fed-batch process

Cyrielle Calmels, Andréa McCann, Laetitia Malphettes, Mikael Rørdam Andersen

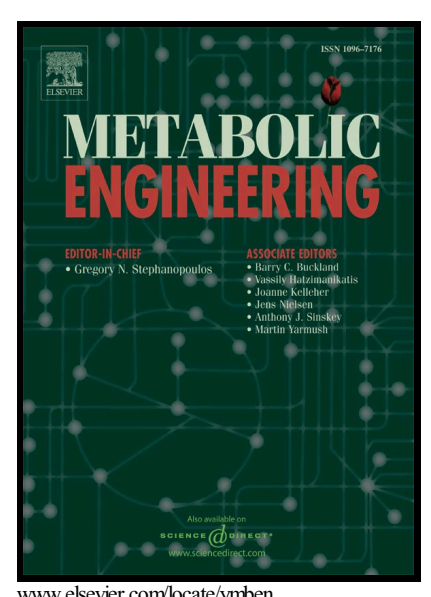

PII: S1096-7176(18)30225-8

DOI: $\quad$ https://doi.org/10.1016/j.ymben.2018.09.009

Reference: YMBEN1470

To appear in: Metabolic Engineering

Received date: 29 June 2018

Revised date: 17 August 2018

Accepted date: 14 September 2018

Cite this article as: Cyrielle Calmels, Andréa McCann, Laetitia Malphettes and Mikael Rørdam Andersen, Application of a curated genome-scale metabolic model of CHO DG44 to an industrial fed-batch process, Metabolic Engineering, https://doi.org/10.1016/j.ymben.2018.09.009

This is a PDF file of an unedited manuscript that has been accepted for publication. As a service to our customers we are providing this early version of the manuscript. The manuscript will undergo copyediting, typesetting, and review of the resulting galley proof before it is published in its final citable form. Please note that during the production process errors may be discovered which could affect the content, and all legal disclaimers that apply to the journal pertain. 


\section{Application of a curated genome-scale metabolic model of CHO DG44 to an industrial fed-batch process}

Cyrielle Calmels $^{\mathrm{a}, \mathrm{b}}$, Andréa McCann ${ }^{\mathrm{a}, \mathrm{c}}$, Laetitia Malphettes ${ }^{\mathrm{a}}$, Mikael Rørdam Andersen $^{\mathrm{b},{ }^{*}}$

${ }^{a}$ Department of upstream process sciences, UCB Pharma, Chemin du Foriest 1, 1420 Braine-l'Alleud, Belgium

${ }^{\mathrm{b}}$ Department of Biotechnology and Biomedicine, Technical University of Denmark, Søltofts Plads 223, 2800 Kgs. Lyngby, Denmark

${ }^{c}$ Mass Spectrometry Laboratory, University of Liège, Allée du six aout, Liège

* Corresponding author E-mail address: mr@bio.dtu.dk

\section{Abstract}

$\mathrm{CHO}$ cells have become the favorite expression system for large scale production of complex biopharmaceuticals. However, industrial strategies for upstream process development are based on empirical results, due to a lack of fundamental understanding of intracellular activities. Genome scale models of $\mathrm{CHO}$ cells have been reconstructed to provide an economical way of analyzing and interpreting largeomics datasets, since they add cellular context to the data. Here the most recently available CHO-DG44 genome-scale specific model was manually curated and tailored to the metabolic profile of cell lines used for industrial protein production, by modifying 601 reactions. Generic changes were applied to simplify the model and cope with missing constraints related to regulatory effects as well as thermodynamic and osmotic forces. Cell line specific changes were related to the metabolism of high yielding production cell lines. The model was semiconstrained with 24 metabolites measured on a daily basis in $n=4$ independent industrial $2 \mathrm{~L}$ fed batch cell culture processes for a therapeutic antibody production.

This study is the first adaptation of a genome scale model for $\mathrm{CHO}$ cells to an industrial process, that successfully predicted cell phenotype. The tailored model predicted accurately both the exometabolomics data ( $r^{2} \geq 0.8$ for $96 \%$ of the considered metabolites) and growth rate $\left(r^{2}=0.91\right)$ of the industrial cell line. Flux distributions at different days of the process were analyzed for validation and suggestion of strategies for medium optimization. This study shows how to adapt a genome scale model to an industrial process and sheds light on the metabolic specificities of a high production process. The curated genome scale model is a great tool to gain insights into intracellular fluxes and to 
identify possible bottlenecks impacting cell performances during production process. The general use of genome scale models for modeling industrial recombinant cell lines is a long-term investment that will highly benefit process development and speed up time to market.

\section{Abbreviations:}

CHO, Chinese Hamster Ovary; GSM, genome-scale metabolic model; pFBA, parsimonious flux balance analysis; GMP, Good Manufacturing Practices; FBA, flux balance analysis; PPP, pentose phosphate pathway; TCA, tricarboxylic acid cycle

\section{Keywords:}

Genome-scale metabolic model; Chinese Hamster Ovary; Flux distribution; Model curation; Metabolic engineering

\section{Introduction}

Chinese Hamster Ovary $(\mathrm{CHO})$ cells are the preferred hosts for the production of recombinant therapeutics proteins, especially as they are able to perform post-translational modifications similar to those carried by endogenous human proteins, which reduces the risk of immunogenicity or low efficacy of therapeutic proteins (Jefferis, 2016). Productivity achieved with mammalian cell culture processes has increased in the last years with vector engineering, cell line development, process conditions and media optimization (Cacciatore et al., 2010; De Jesus and Wurm, 2011). However, the time spent to adapt the best process conditions and feeding strategy to a new industrial recombinant cell line still remains significant, as time-consuming empirical trials are the main reliable strategy for achieving high-yielding processes As production of GMP material is on the critical path to first-in-man, accelerating the development and optimization of biopharmaceutical production has a direct impact on the availability of the active pharmaceutical ingredient for patients. Furthermore, the industry needs to increase process yields while keeping high quality standards to make the drug affordable for the health systems and ensuring access for patients (Li et al., 2014).

Moreover, to avoid any misincorporation in proteins due to depletion of amino acids (Khetan et al., 2010; Wen et al., 2009), medium optimization studies often lead to fed-batch culture conditions where essential amino acids are not depleted during the process, and most of non-essential amino acids are provided in sufficient quantities so that the cells can consume amino acids at maximal rates without exhaustion in the medium (Reinhart et al., 2015). Exception has to be made for amino acids whose catabolism generates toxic by-products, such as glutamine catabolized into glutamate and ammonium (Chen and Harcum, 2005). Thus, a detailed analysis of CHO cell metabolism is required to adapt feeding strategies 
and cell culture media. It will efficiently identify bottlenecks that could explain suboptimal amino acid catabolism experimentally observed during the process, thus resulting in improved cell growth, viability and process yields.

In order to enable such a fast and detailed metabolic analysis of production CHO cells, a consensus genome scale model (GSM) and cell linespecific stoichiometric models have been recently reconstructed by a community of researchers (Hefzi et al., 2016). These models describe the reaction network, accounting for all the known $\mathrm{CHO}$-specific conversions of substrates into metabolic products and biomass components. $\mathrm{CHO}$ specific genome-scale metabolic models are built based on genomic and physiological information in a specific organism (Calmels et al., accepted). With the integration of a linear based programming framework, GSM can be used to compute enzymatic rates under defined environmental conditions. CHO GSM enables in silico prediction of the effect of gene deletions, gene over-/under-expression, the possibility for identification of metabolic targets to reduce toxic by-product formation, and the combination of other omics-data types in a single computational framework (Kaas et al., 2014).

In this study, a cell line-specific model for CHO DG44 was developed as a support tool for interpretation of experimental data, in order to understand better the pathways underlying cell growth and protein production in an industrially relevant cell culture process. The predicted power of the initial model was improved by manual curation of the model. The curated model was tested with an industrial fed batch process of a cell line producing a monoclonal antibody, by computing the daily flux distribution of the cells throughout the bioreactor production step.

\section{Material and methods}

\subsection{Cell culture and extracellular metabolite analysis}

\subsubsection{Cell culture conditions}

4 fed batch cell culture experiments were run independently, for which cell phenotype, variation of extracellular metabolite concentrations, and process parameters were monitored. A CHO DG44 cell line producing mAb1 was cultivated in $2 \mathrm{~L}$ stirred tank glass bioreactors, controlled by supply towers (C-DCUII, Sartorius Stedim Biotech) and monitored by a multi-fermenter control system (MFCS, Sartorius). The cells were growing under serum free conditions in a proprietary and chemically defined media. Precultures were cultivated in increased volume capacity of Erlenmeyer flasks (Corning Inc, Germany) on a shaking device at $37^{\circ} \mathrm{C}$ and $5 \% \mathrm{O}_{2}$ in a humid atmosphere. The starting culture volume was 


\section{ACCEPTED MANUSCRIPT}

identical for the different production run and the bioreactors were inoculated at similar target seeding density. The cultivation temperature was kept constant at $36.8^{\circ} \mathrm{C}$ and the impeller used was a 3-segment blade impeller. During the cultivation, the pH was fixed at 7.0, with an allowable variation of 0.2 , and controlled by gassing $\mathrm{CO}_{2}$ and a sodium carbonate solution. Dissolved oxygen was maintained at $40 \%$ of the saturation concentration. Continuous nutrient feeding was started 72 hours after inoculation, with predetermined rate using a proprietary, chemically defined concentrated feed. The feed rate was adapted every day, following a predefined feeding profile. In addition to this continuous feed, a bolus feed addition also started 72 hours after inoculation. Samples were taken once a day, before feeding. When the glucose concentration was below $5.6 \mathrm{~g} / \mathrm{L}$, a glucose solution of $500 \mathrm{~g} / \mathrm{L}$ was added as a bolus. Specific growth rate was calculated for each experimental condition as in equation 1:

$$
\mu=\frac{\Delta \ln (V C C)}{\Delta t}
$$

The exponential growth equation is described by equation 2 :

$$
\ln (X)=\ln \left(X_{0}\right)+\mu t
$$

where $\mu$ is the specific growth rate, $X$ is the cell density (i.e. cell mass or number per unit volume of culture medium), $t$ is time, and $X_{0}$ is the initial cell density at the onset of exponential growth. This equation was used to determine the specific growth rate from linear regression of cell density measurements over time.

\subsubsection{Analytical methods}

Samples from the bioreactor were taken daily for cell density and viability analysis using the VI-CELL ${ }^{\circledR}$ XR (Beckman-Coulter, Inc., Brea, CA), based on the trypan blue exclusion method. Samples were centrifuged and supernatants were analyzed to quantify concentration of glucose, lactate, amino acid and monoclonal antibody. Glucose, lactate, glutamate, glutamine and ammonium concentrations were determined using a Cedex Bio HT Analyzer (Innovatis, Bielefeld, Germany) or a NOVA 400 BioProfile automated analyzer (Nova Biomedical, Waltham, MA). Cell culture supernatant samples were stored at $-80^{\circ} \mathrm{C}$ or directly analyzed for product titer with a ForteBio Octet model analyzer (ForteBio, Inc., Menlo Park, CA) or protein A high performance liquid chromatography (HPLC). Amino acids were analyzed by reversed-phase UPLC (Waters AccQ - Tagultra method) after ultra-filtration using Amicon Ultra- $0.5 \mathrm{~mL}$ centrifugal filters (Merck Millipore, Billerica, MA). pH and DO were measured on-line and the measurement accuracy was verified through offline analysis of $\mathrm{pH}$ and partial pressure of $\mathrm{CO} 2$ (pCO2) using a BioProfile $\mathrm{pHOx}^{\circledR}$ blood gas 


\section{ACCEPTED MANUSCRIPT}

analyzer (Nova Biomedical Corporation, Waltham, MA). Cellular lipids were extracted based on Matyash method (Matyash et al., 2008), separated by hydrophilic interaction liquid chromatography (HILIC) and detected by triple quadrupole mass spectrometry (Waters, Millford, MA). Internal ${ }^{13} \mathrm{C}$ labelled lipids standards provided by Avanti Polar Lipids (Alabaster, $\mathrm{AL}$ ) were added prior to extraction to ensure an accurate quantification.

\subsection{Modeling procedure}

\subsubsection{Genome scale model used}

A community genome scale metabolic network was recently reconstructed by different research groups (Hefzi et al., 2016), first by combining two established genome scale reconstructions for Homo Sapiens (Recon 1 (Duarte et al., 2007) and Recon 2 (Quek et al., 2014)) and then by manual curation to find CHO homologs to human genes (Hefzi et al., 2016). Genes that are specifically expressed in CHO DG44 cell lines were identified and selected to generate a cell line specific model for CHO DG44, based on existing data used as input information in the Gene Inactivity Moderated by Metabolism and Expression (GIMME) algorithm (Becker and Palsson, 2008).

For each cell line modeled, the reaction responsible for antibody production in the model was tailored to the antibody produced by the cell line chosen. The stoichiometric coefficients of the amino acids required to produce the $\lg$ Gere changed to the one corresponding to the specific amino acid sequence of the antibody produced, thus generating a genome scale model for the cell line producing mAb1.

\subsubsection{Theoretical approach for modeling}

The modeling framework can be represented by a stoichiometric matrix $(S)$ and a vector of reaction fluxes $(v)$ indicating the reaction rates. The basic steady state mass balance constraint can be enforced by the linear equation 5 . Additional constraints can be introduced by restricting fluxes with upper or lower bounds through inequality 6. To find the flux distribution, parsimonious enzyme usage Flux Balance Analysis (pFBA) (Lewis et al., 2010) was employed. This optimization method is based on the assumption that the cell is using a minimum amount of enzymes to reach a maximized objective, under the hypothesis of steady state. The mathematical formulation of the objective function is given by equation 3 , and the requirement for the minimum absolute values among all the alternatives optima is described by equation 4 . To find the flux 
distributions, the network is constrained by imposing lower and upper bounds for each flux, and by assuming a steady state condition, which leads to the following optimization problem:

$\max c v$
$\min \sum|v|$
s.t. $S v=0$
and $v \min \leq v \leq v \max$

Where $c v$ corresponds to the objective function and $c$ is a vector of weights, indicating how much each reaction contributes to the objective function.

Linear programming was performed using Gurobi Optimizer (Gurobi Optimization Inc., Houston TX) in Python 2.7.12 (Python Software Foudation, Delaware, United States).

To represent experimental cell growth conditions at each day of the process, which vary in particular because of feed additions and by-product secretion, a maximal bound was set equal to the experimental flux measured for 24 external metabolite rates. Thereby, if the predicted values were comparable to the experimental, we could consider that the distribution solution was worth being analyzed. The calibration of the model was performed by applying pFBA to successive points in cell culture time (day 2 to 7 ) with different constraints related to medium's components consumption/production rates. Daily experimental rates of 24 metabolites in medium were calculated from experimental measurements, postprocessed with a smoother function in order to reduce experimental noise.

\subsection{Data processing and transforming primary data into flux constraints}

Substrate concentrations at each day of the cell culture were transformed into rates in mmol of product per gDW of cells per hour. The modeling time frame chosen was between day 2 and day 7 of cell culture, which correspond to the exponential growth phase where the pseudo-state is assumed. All the input flux rate values were calculated using an average dry cell weight of $\mathrm{CHO}$ cells as $330 \mathrm{pg} / \mathrm{cell}$, as a literature average value (Bonarius et al., 1996; Vriezen, 1998; Xie and Wang, 1994; Zupke and Stephanopoulos, 1995). Daily experimental uptake or production rates of 


\section{ACCEPTED MANUSCRIPT}

24 metabolites in medium were calculated from experimental measurements, post-processed with a smoother function in order to reduce experimental noise and normalized with one value corresponding to a specific growth rate of the mAb 1 producing cell line. The experimental variation can be explained by several factors, including the sensibility and technical variability of the analytical quantification method, but also the degree of biological reproducibility inter-process.

Experimental production and consumption rates calculated were smoothed using a kernel smoother statistical function, in order to avoid irregular data points and noisy observations obtained from experimental results.

Given a random sample $\left(x_{i}, y_{i}\right) \in R^{2}, i=1, \ldots n$, with a continuous, univariate density $f$, the kernel function or weighting function $K \lambda(x)$ assigns a weight to xi based on its distance from each query point $x$. The kernels $K \lambda$ are typically indexed by a parameter $\lambda$ that dictates the width of the neighborhood. For a given scale parameter $\lambda$, the weight sequence is defined by the equation below (Hastie et al., 2009) :

$K \lambda(x)=\sum_{i=1}^{n} \frac{K\left(\frac{x-x i}{\lambda}\right)}{\sum_{i=1}^{n} K\left(\frac{x-x i}{\lambda}\right)} y i(7)$

The Kernel smoother method was applied on calculated experimental flux rates with the support of the statistical software JMP. Suppl. Fig. 1 displays the example of arginine which exhibits relatively good correlation coefficients after smoothing. Smoothed experimental values were considered as the more accurate approximation to model cell metabolism due to experimental noise.

The calculated and smoothed experimental values were then used to set the upper or lower limit of the consumption or production rate of the metabolites measured daily in the extracellular environment. Exception was made for ammonium, alanine, and lactate that were set strictly equal to the experimental value in order to constrain the model with the metabolic switch between consumption and production.

The final set of constrained metabolite exchanges comprised the uptake and secretion of 24 metabolites. The limitations for uptake and production rates were set for each day of the time frame chosen for modeling the cell culture process. The objective function chosen was maximization of growth. Estimation of metabolic fluxes was performed using CobraPy (Ebrahim et al., 2013).

\subsection{Statistical analysis}




\section{ACCEPTED MANUSCRIPT}

For evaluating significant differences between means of datasets with replicates in different conditions, a one-way ANOVA was performed. When significant, post hoc analysis was performed using Tukey's honestly significant difference (HSD) to group data by means. All tests were performed using JMP Software (SAS Institute Japan Ltd., Japan) and GraphPad Prism software (Version 7.02, Graphpad Software, San Diego, CA).

\section{Results and discussion}

\subsection{Manual curation}

The recent publication of the most complete genome scale model for CHO cells (Hefzi et al., 2016) has offered the industrial world a chance to examine metabolic fluxes of production cell lines in different pathways and under distinct culture conditions. In bioprocess development cell cultures, $\mathrm{CHO}$ cells exhibit a specific metabolic profile characterized in particular by elevated stress (Templeton et al., 2013). Thus to improve quality of predictions, the initial model was adapted to a specific recombinant production cell line. Three types of modifications were required to tailor the generic model to UCB's recombinant production cell line: (i) removal of metabolic reactions from the network structure, as their activation relied on unknown parameters based on thermodynamic and chemical constraints, or more general scientific knowledge, (ii) addition of metabolic reactions either to complete the central carbon metabolism as described in Cricetulus griseus, or to adapt the genome scale model to the metabolism of high yielding production cells, and (iii) change of reaction parameters in order to constrain the network as much as possible according to literature, to obtain more biologically meaningful results. A total number of 601 reactions were modified to produce the tailored genome scale model as detailed in the supplementary excel file. A first technical section describes modifications which aim at curating the model to make it generally more functional with the available constraints, a second section gathers modifications that were observed as being specifically related to the metabolism of high producers. 


\subsubsection{General modifications of the metabolic network made to increase prediction performances}

\subsubsection{Deletion of extracellular amino acid co-transporters}

Initial modeling trials with Flux Balance Analysis on the generic DG44 model described infeasible solutions composed of isolated activated fluxes, predicted to occur at the maximum upper limit of the supposedly unconstrained reactions, as it is common to choose an arbitrarily elevated number to represent unconstrained fluxes $(1000 \mathrm{mmol} / \mathrm{gDW} / \mathrm{h})$. Several actions were undertaken in order to increase the predictive power of the initial model. The first action was to change directionality of reactions that import nutrients to lock them in one direction, in order to uniquely allow consumption from the medium into the cell. The second action was the choice of a most suitable mathematical approach, namely Parsimonious FBA, as this method has been shown in previous studies to avoid degeneracy of FBA solutions (Machado and Herrgard, 2014; Toroghi et al., 2016). pFBA relies on the same principles as FBA, with an additional constraint on minimizing the sum of total fluxes across the entire network (Lewis et al., 2010). The third action was taken after the main source of highly activated fluxes remaining in all the computed solutions was identified at the level of amino acid transportation.

Indeed, as the model accurately represents mammalian cell composition, it contains all known types of amino acid transporters which are assigned to a large number of different families. However in vivo the activation of one type over the other will mainly depend on dynamic parameters that are not known and/or not included in the model. The constraints that are not considered in the model fall into the following categories: substrate concentration gradient; maintenance of the electrochemical gradient, that may vary with movements of charged cotransporters across membranes; charge, size and hydrophobicity of the molecules; flow regulation in response to binding of signaling molecule; balance of osmotic pressure; regulation of intracellular $\mathrm{pH}$; degree of saturation of the transporters; preferential substrates; interdependence of secondary and tertiary active transporters, or inhibition/stimulation mechanisms which are not even well understood for all the transporters (2002; GM., 2000). The complexity of the model was thus dramatically reduced by silencing 537 transporters (Supplementary excel file of all reactions modified). No more inconsistencies of solutions containing loops characterized by high fluxes feeding each other were observed (Thiele and Palsson, 2010). This removal of the transporters led to disappearance of infeasible cycles linked to amino acid uptake fluxes.

\subsubsection{Manual curation to simplify mathematical modeling}


The initial metabolic network contains identical reactions allocated to different cellular compartments, which is the case e.g. for the reaction catalyzed by fumarase as illustrated in Suppl. Fig. 2. A literature research was conducted and the reaction has been shown to be mainly localized in mitochondria (Bowes et al., 2007), so the cytosolic version of the reaction was removed from the model.

We observed that in some cases identical reactions allocated to different compartments could be activated together at high rates. This can be visually identified in predictive flux distributions with the presence of internal cycles, defined by their capacity of revolving independently of the rest of the network. The substrates of one reaction correspond to the products of the other across compartments, while using metabolites that the cell is typically producing in excess (such as water, protons, sodium, present in numerous reactions in the model) or other metabolites which synthesis would require valuable co-factors such as NADPH or ATP. Consequently, these internal cycles do not only involve the identified couple of reactions but have an impact on the entire set of solution, leading to errors in the estimation of the objective function and redox balancing (Zomorrodi et al., 2012).

Similarly, we identified reactions using either NADPH or NADH as cofactor that are systematically activated together at high rate in flux distributions. The flux solution computed activation in high rates of these paired reactions containing similar substrates and products, using NADPH as coenzyme in one direction and NADH in the opposite direction. As a matter of fact, a few enzymes can use both NADPH/NADH and most show a strong preference for one over the other, for each internal cycle of this type identified, one of the reactions was removed based on a literature and database research to select the true biological reaction to be activated. The removal was thus based on information from BRENDA (Placzek et al., 2017) and KEGG databases, as well as literature (see Supplementary excel file of all reactions modified). If no information could be found it was decided to remove the reaction using NADPH, using the rationale that an NADH-driven reaction is more likely as the total concentration of NAD+/NADH in most tissues is about $10^{-5} \mathrm{M}$, whereas that of NADP+/NADPH is about $10^{-6} \mathrm{M}$ (Blacker and Duchen, 2016 ; Boyle, 2005). The entire modifications resulting from the manual curation are listed in the supplementary excel file.

\subsubsection{Addition of reactions}

The initial genome scale model specific for CHO DG44 cells was extracted from a general consensus model with an algorithm based on gene expression thresholds. As demonstrated recently (Opdam et al., 2017), the algorithm used for extraction and the gene expression threshold are key decision parameters to build a cell line specific model and can significantly impact model content and accuracy. 


\section{ACCEPTED MANUSCRIPT}

In total, seven reactions were manually added (Table 1) to complete glycolysis, oxidative phosphorylation and TCA pathways as compared to the pathways found in the KEGG database (Kanehisa et al., 2017; Kanehisa and Goto, 2000; Kanehisa et al., 2016) for Cricetulus griseus.

A new compartment named mitochondrial membrane $(\mathrm{mm})$ was added in the model. As the reaction catalyzed by succinate dehydrogenase (SDH) is inherent to the activity of complex II of respiratory chain (Cecchini, 2003; Lenaz and Genova, 2010), the reducing power FADH2 produced by SDH and used by complex II was assigned to this new compartment in order to make sure that the FADH 2 produced by the mitochondrial SDH is directly fueled in the electron transport chain. A directly measurable result can be visualized in Suppl. Fig. 3. The predicted activity in complex II was increased in the curated model and the progression over time seems to follow the physiological conditions, if we consider oxygen uptake rate experimentally measured during cell cultures as a marker of energy metabolism (Deshpande and Heinzle, 2004).

Table 1

List of reactions added in the model.

\begin{tabular}{|c|c|c|}
\hline Reaction name & Reaction ID & Reaction \\
\hline Glyceraldehyde-3-phosphate dehydrogenase & GAPD & g3p_c + nad_c + pi_c --> 13dpg_c + h_c + nadh_c \\
\hline Pyruvate kinase & PYK & $a d p \_c+h \_c+p e p \_c-->a t p \_c+p y r \_c$ \\
\hline NADH dehydrogenase, mitochondrial & NADH2_u10m & 5 h_m + nadh_m + q10_m --> 4 h_im + nad_m + q10h2_m \\
\hline Pyruvate dehydrogenase & $\mathrm{PDHm}$ & coa_m + nad_m + pyr_m $\Leftrightarrow=>$ accoa_m + co2_m + nadh_m \\
\hline Glycerol-3-phosphate dehydrogenase & G3PD & dhap_c + nadh_c --> glyc3p_c + nad_c \\
\hline Glutathione peroxidase and catalase, cytosol & GTHP_CAT_c & 2 gthrd_c +3 h2o2_c --> gthox_c +4 h2o_c $+02 \_c$ \\
\hline Glutathione peroxidase and catalase, mitochondria & GTHP_CAT_m & 2 gthrd_m +3 h2o2_m --> gthox_m +4 h2o_m $+02 \_m$ \\
\hline
\end{tabular}

3.1.2. Modifications of the metabolic network directly related to high yielding production cells metabolism 


\section{ACCEPTED MANUSCRIPT}

\subsubsection{Correction for activation of the oxidative stress pathway}

Industrial production cell lines are subjected to elevated oxygen levels, and this highly activated aerobic cell metabolism generates continuously free radical species that can impact cell performances and product quality (Ha et al., 2018; Selvarasu et al., 2012; Templeton et al., 2013). To tailor the network to this specific metabolic trait related to high yielding production cells, the following modifications were applied: (i) deletion of the reaction for $\mathrm{H}_{2} \mathrm{O}_{2}$ secretion; hydrogen peroxide being an unstable molecule, it quickly reacts with any present metabolite, so any significant secretion cannot be representative of the biological reality, (ii) conservation of the cytochrome $\mathrm{C}$ oxidase reaction producing $2 \%$ of superoxide to have a constant production of reactive oxygen species associated to cellular respiration (Turrens, 2003), (iii) a simplified version of the antioxidant defense system was included by fusing the catalase and glutathione peroxidase reactions, as illustrated in Fig. 1 , in order to have them activated simultaneously when computing the solution. This global detoxifying reaction called "GTHP_CAT" was created as a balanced equation of the two main antioxidant enzymes catalase and glutathione peroxidase (Weydert and Cullen, 2010), localized both in mitochondria and cytosol (Table 2).

These modifications represent a major improvement in the model, as it allows activation of glutathione peroxidase reaction, and can be used to have a more representative use of the substrates and products involved in regulation of oxidative stress. However, the global rate of the reaction is only comparable to in vivo detoxification rates if there is a fixed ratio between catalase and glutathione peroxidase. At high $\mathrm{H} 2 \mathrm{O} 2$ levels, catalase has been shown to have a prominent role in mammalian cells (Ho et al., 2004) with an activity almost 100 times higher than glutathione peroxidase quantified in $\mathrm{CHO}$ cells (Keizer et al., 1988). However in our knowledge this ratio has been shown as constant during cell culture, and may be subject to variations. Overall, the modifications made allow a more precise prediction of the redox and antioxidant status of the cell at different days of the cell culture. A more realistic NADPH/NADP ${ }^{+}$requirement can be computed, so the model could also be used to define a strategy for minimization of biological damages caused by oxygen-derived radicals. 


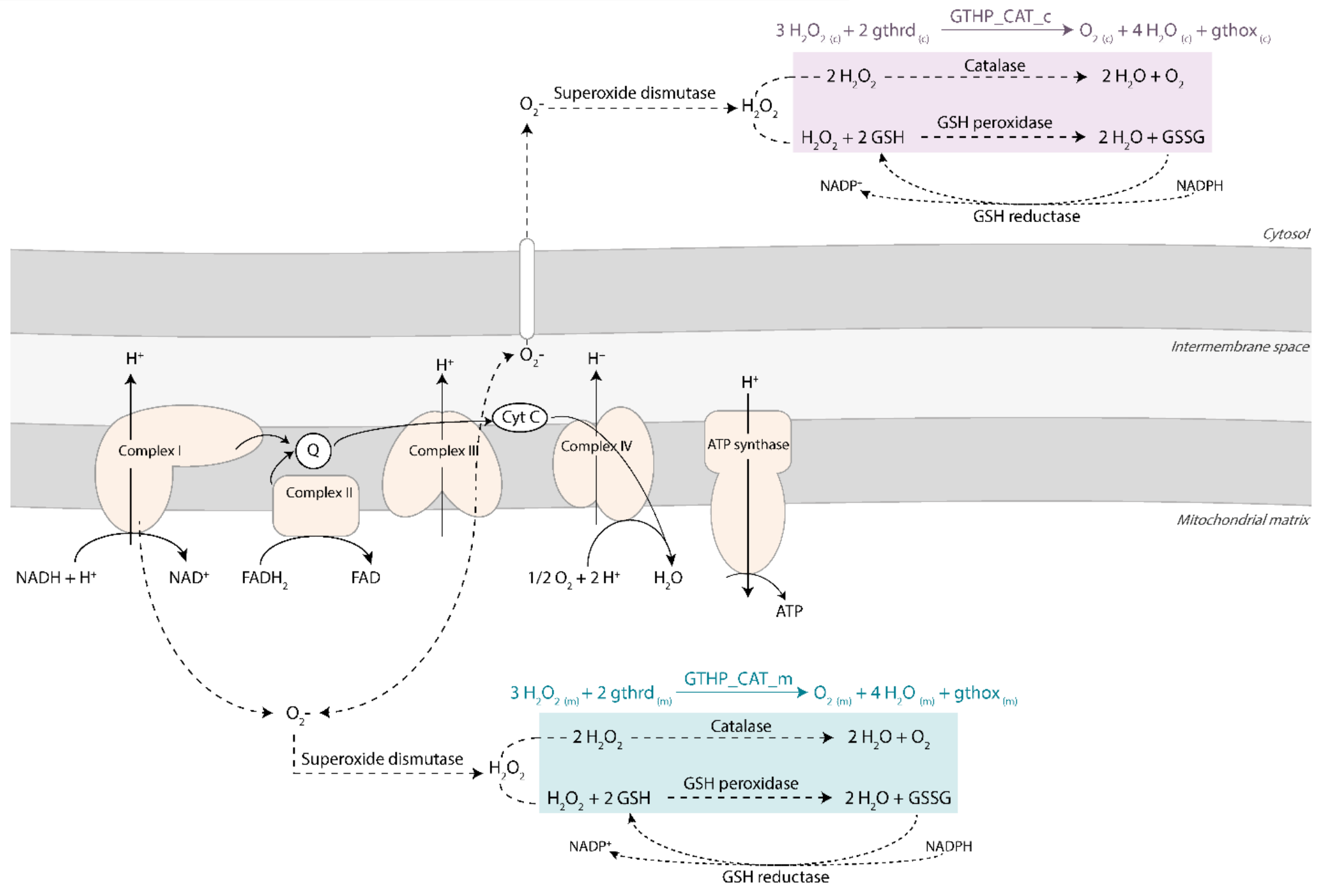

Fig. 1. Pathways for generation of ROS by mitochondrial electron transport chain, included in the model. The balanced equations given by the addition of the reactions catalyzed by the enzymes catalase and GHS peroxidase were added in the model, named GTHP_CAT_m and GTHP_CAT_c, both in the mitochondrial and cytosolic compartment. Plain line : oxidative phosphorylation included in the initial model ; Dash lines : oxidative stress included in the initial model ; GSH and gthrd : reduced glutathione; GSSG and gthox : oxidized glutathione.

\subsubsection{Mathematical compensation for lipid accumulation}

Lipidomics data we generated (Suppl. Fig. 4) and recently published studies (Ali et al., 2018) have demonstrated the importance of lipid metabolism for the phenotype of $\mathrm{CHO}$ cells cultivated in fed-batch production, which could also explain a large cell diameter observed with high producers (Pan et al., 2017). There is experimental evidence that lipids accumulate in the cells towards the end of the process, especially glycerophosphatidylinositol and triacylglycerol. For mammalian cells, this storage mechanism gives a competitive evolutionary advantage in case of fluctuations in the composition of the extracellular medium, since they are able to accumulate lipid droplets to form a source of energy 
storage (Farese and Walther, 2009). These lipid droplets are made of a phospholipid monolayer surrounding sterol esters and triacylglycerols, and their growth seems to be associated to triacylglycerol synthesis. This information was included in the model with the addition of a constraint on minimum production rate of triglyceride, and no strict constraints was fixed for lactate secretion. ANOVA found significant difference between the 2 modeling conditions - considering and excluding triacylglycerol accumulation - from day 3 to day 0 included $(p<0.0003)$. In Fig. 2, pairwise comparisons were performed using Tukey's HSD test with a family-wise confidence level of 95\%. Flux rates extracting statistically similar values were grouped and labeled accordingly. In the model, adding a constraint to consider a storage of triglycerides had a direct impact on predictive lactate production rate, which was quantitatively comparable to measured rates without subverting the flux rate distribution solution. In this regard, the tailored genome scale model is accurately representative of the metabolism of high producing cells and can even be considered to be used as a complementary tool for the cell line selection process.

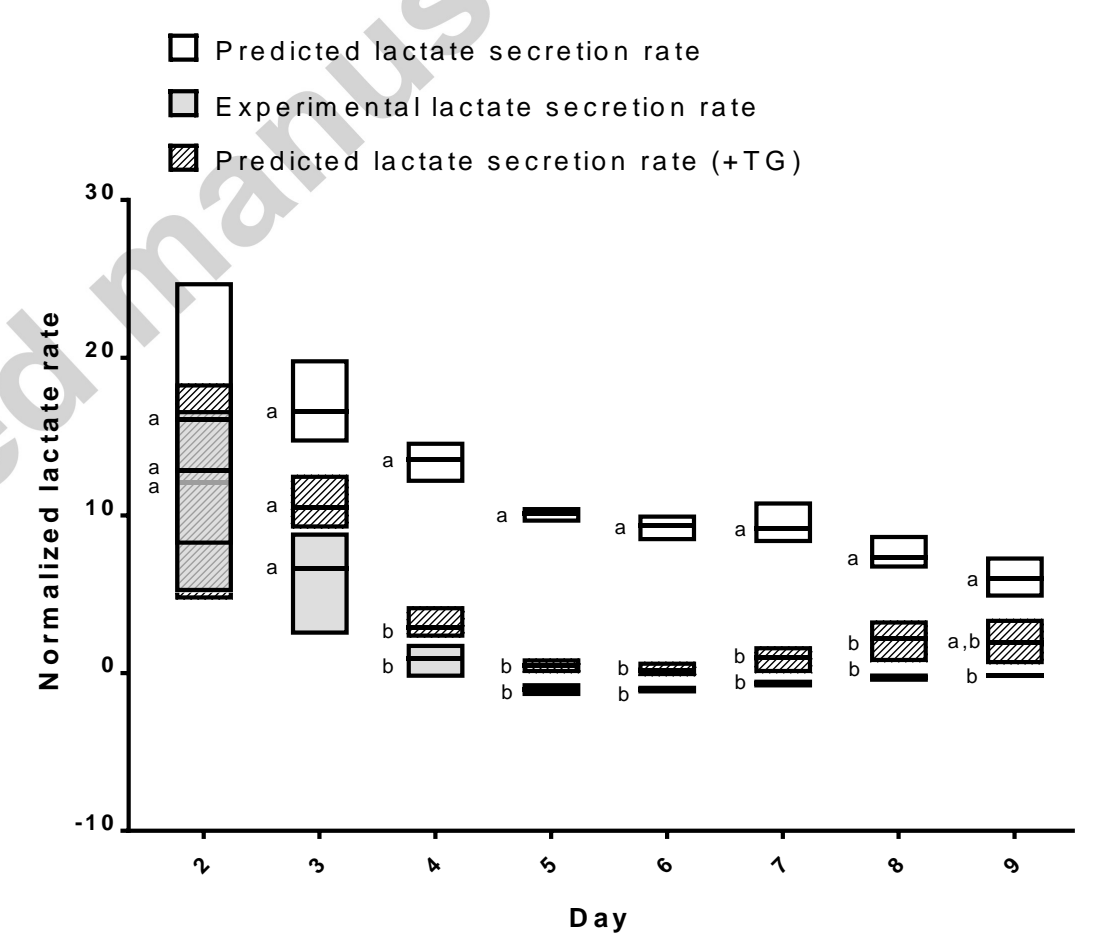

Fig. 2. Impact on the addition of predicted lipid accumulation on predicted lactate flux rate. The experimental data correspond to control processes of cell line CHO DG44_mAb $1(n=3)$ and was computed independently to obtain the mean and standard deviation given when modeling using the genome scale model. The same upper and lower bounds were set each day in the two predictions. Label a is for experimental condition, $b$ is predicted values. The prediction value corresponding to predicted lactate secretion rate (+ TG) has an additional constraint of a minimal daily triglyceride "accumulation" rate (lower bound set for "EX_tag_cho_e_" reaction). Values are normalized according to growth rate across all datasets. Conditions showing a statistical similar lactate flux rate were grouped together (group a or b) based on Tukey's HSD (P < 0.05). 


\subsubsection{Summary of the curation process}

The final model consists of 3942 reactions, 2750 metabolites and 1188 genes as described in Table 2 . The initial model was simplified in order to match with the mathematical approach used and the available set of constraints applied. Moreover, the model was adapted to high yielding cell metabolism and can be used to infer more knowledge about industrial processes. A pathway for oxidative stress was specifically designed so that the impact of high oxygen concentration in cell culture process is more accurately represented. This addition in the model makes possible to simulate cell adaptation to oxidative stress, by increasing levels of antioxidant defenses and by metabolizing larger amount of glucose via the pentose phosphate pathway (Ahn and Antoniewicz, 2011). The role of lipid storage during cell culture was also included, which makes a difference in the predictions in terms of flux distribution and correlates with in vivo observations. Considering lipid metabolism in cell culture with the help of a genome scale model is one of the most adequate way of doing so, as lipid metabolism needs to be put in perspective of the entire metabolic network: Lipids can have an impact at different metabolic levels on energy storage, nutrient intake, cell growth, and regulation of cellular processes. Physiological features characterizing specifically high producing cell lines, such as those added in the curated genome scale model, are important to capture accurate phenotypic predictions. Other important features can be considered in future work for enhancing predictive power, such as the incorporation of a more complex amino acid import processes. In a recent study, 16 amino acid transporters have been identified in abundance in high-producing recombinant cell lines, with a regulated coordinated antiport system to supply amino acids, and variations in relative expression levels of transporters depending both on the metabolism of the engineered $\mathrm{CHO}$ cell line and on amino acid concentration in cell culture medium (Geoghegan et al., 2018). Prediction of energy utilization and specific productivity could also be improved by considering the level of difficulty associated to the folding step of a specific antibody (Le Fourn et al., 2014), as well as the protein secretory pathway which has been identified as a bottleneck in some cases (Pieper et al., 2017; Rahimpour et al., 2013).

The resulting model is tailored to industrial cell lines and can be used with simple information as input, by adding constraints available from daily metabolite concentration measurements in the culture medium.

\section{Table 2}

Comparison of the original and curated model. 
C. Calmels et al. / Metabolic Engineering

ACCEPTED MANUSCRIPT

\begin{tabular}{|c|c|c|}
\cline { 2 - 3 } \multicolumn{1}{c|}{} & Initial model & Curated model \\
\hline Number of reactions & 4533 & 3942 \\
\hline Amino acid metabolism & 205 & 196 \\
\hline Carbohydrate metabolism & 181 & 175 \\
\hline Cofactor and vitamins & 152 & 146 \\
\hline Nucleotide metabolism & 207 & 203 \\
\hline Lipid metabolism & 570 & 565 \\
\hline Energy metabolism & 21 & 21 \\
\hline Transporters & 2616 & 2072 \\
\hline Glycan synthesis & 142 & 142 \\
\hline Other & 422 & 439 \\
\hline Number of metabolites & 2750 & 2750 \\
\hline Number of genes & 1132 & 1188 \\
\hline
\end{tabular}




\section{ACCEPTED MANUSCRIPT}

3.2. Application of the curated model to one industrial cell line during fed batch production

In order to validate the manual curation performed, the model was tested for simulation of 4 independent $2 \mathrm{~L}$ cell culture bioreactor run from a known production process. The evaluation of the curated tailored model was based on the comparison of cell performances and exometabolomics data with experimental data, and with knowledge available in literature.

\subsubsection{The predicted phenotype of the industrial recombinant producing cell line is comparable with experimental observations}

The cell count over time for the culture of a CHO DG44 cell line producing mAb1 grown in supplemented medium is presented in Suppl. Fig. 5. This plot distinguishes a first period, from inoculation to day 7 , where cell growth dominates the exponential phase. This time frame was selected for modeling, as it was considered to be a key phase in cell culture. During this period, cell counts and antibody titer increase respectively approximately 13- and 11-fold. Experimental specific growth rate decreases and specific productivity increases linearly from day 2 to day 7. Therefore, during this time frame, most of the amino acid demands can be attributed to both cell proliferation and maintenance, as well as product assembly for antibody production.

As daily sampling was performed before media additions, it was assumed that perturbations of metabolic steady state due to media additions were negligible in a time frame of 20-24 hours (Segre et al., 2002). This time frame can be considered to be sufficient to achieve equilibration of extra and intracellular equilibration of metabolites, thus to attain stationary values of macroscopic properties of the bioreactor (cell density and metabolite concentrations). pFBA was applied from day 2 to day 7 of cell culture processes, when experimental specific growth rate shows a linear evolution over time and cell death is negligible. After day 7, cell death becomes more important than cell growth, which currently cannot be predicted well by the model.

\subsubsection{The majority of metabolite secretion and production rates are predicted accurately by the tailored model}

The model was semi-constrained with exometabolomics data and the four predictions were computed for each independent $2 \mathrm{~L}$ run. $\mathrm{By}$ doing so the goal was to verify that the biosynthetic growth requirements were comparable to the in vivo metabolic needs, and to some extend this semi loose constraints were applied to calibrate the model with the available nutrients in the experimental growth environment. 


\subsubsection{Prediction of amino acid utilization}

As amino acids are one of the most important set of metabolites for growth of mammalian cells and production of proteins, we wanted to see how the model predicts their uptake and production.

Fig. 3 shows the comparison between the predicted and the experimental value of the measured metabolites. Correlation coefficient is 0.91 for predicted growth rate, which indicates a very reliable prediction when applying the tailored model to high yielding cells. Simulations were also performed with changing the objective function to maximization of antibody production from day 6 to 9 , and in this case correlation coefficient of 0.61 indicates inaccurate predictions compared to experimental specific productivity. This inaccuracy can be explained by the fact that cells keep growing by biomass during exponential phase and also during the stationary phase (Pan et al., 2017), thus the most appropriate objective function to be applied during the chosen time frame is maximization of biomass production.

Among various amino acids, the highest consumption rates were observed for asparagine and serine both in vivo and in silico. High asparagine consumption is linked to increased alanine, glycine and ammonia concentration during the process (Hansen and Emborg, 1994; Selvarasu et al., 2012). Predicted consumption rates of the primary carbon sources, glucose and glutamine (Burgess, 2011), are strictly equal to the experimental rate. At the early stage of the exponential phase, cells exhibit a high glucose consumption rate and a high lactate production resulting from a high glycolytic rate. This is consistent with the Warburg effect, which is observed in culture of continuous cell lines under aerobic conditions and describes a phenotype characteristic of tumor cell metabolism (Deberardinis et al., 2008). Ammonia secretion rate resulting from glutamine consumption in the initial exponential phase significantly decreased after day 3 , both for the predicted and experimental values. As shown in Fig. 3. B., $96 \%$ of the considered metabolites showed a correlation coefficient higher or equal to 0.8 with experimental value. In this regard, the methodology applied for modeling reflects accurately the cellular metabolism in early exponential phase, at the level of nutrient source utilization and cell performances. 
A

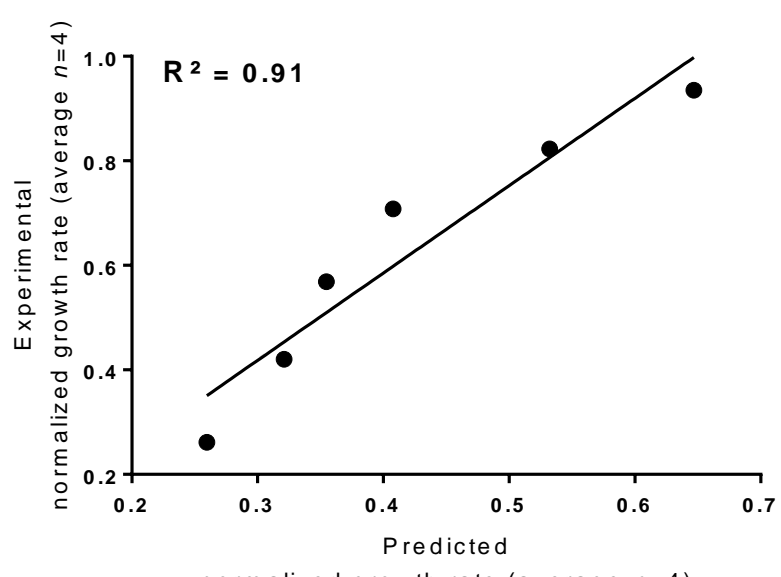

normalized growth rate (average $n=4$ )

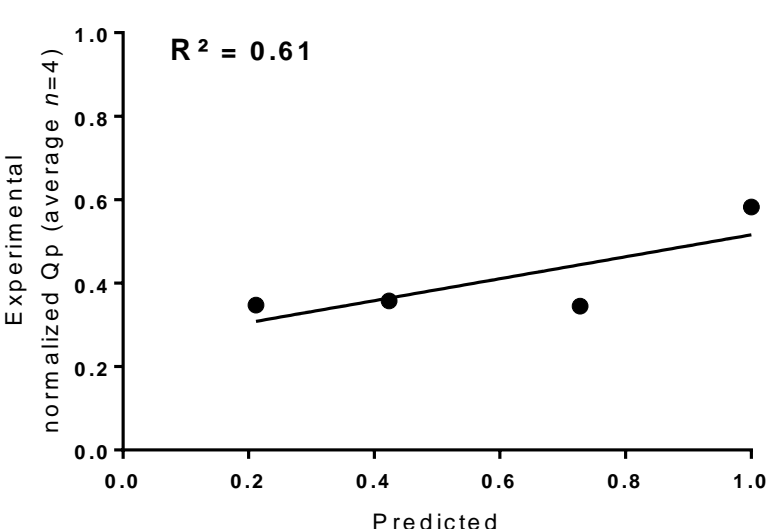

normalized Qp (average $n=4$ )

B

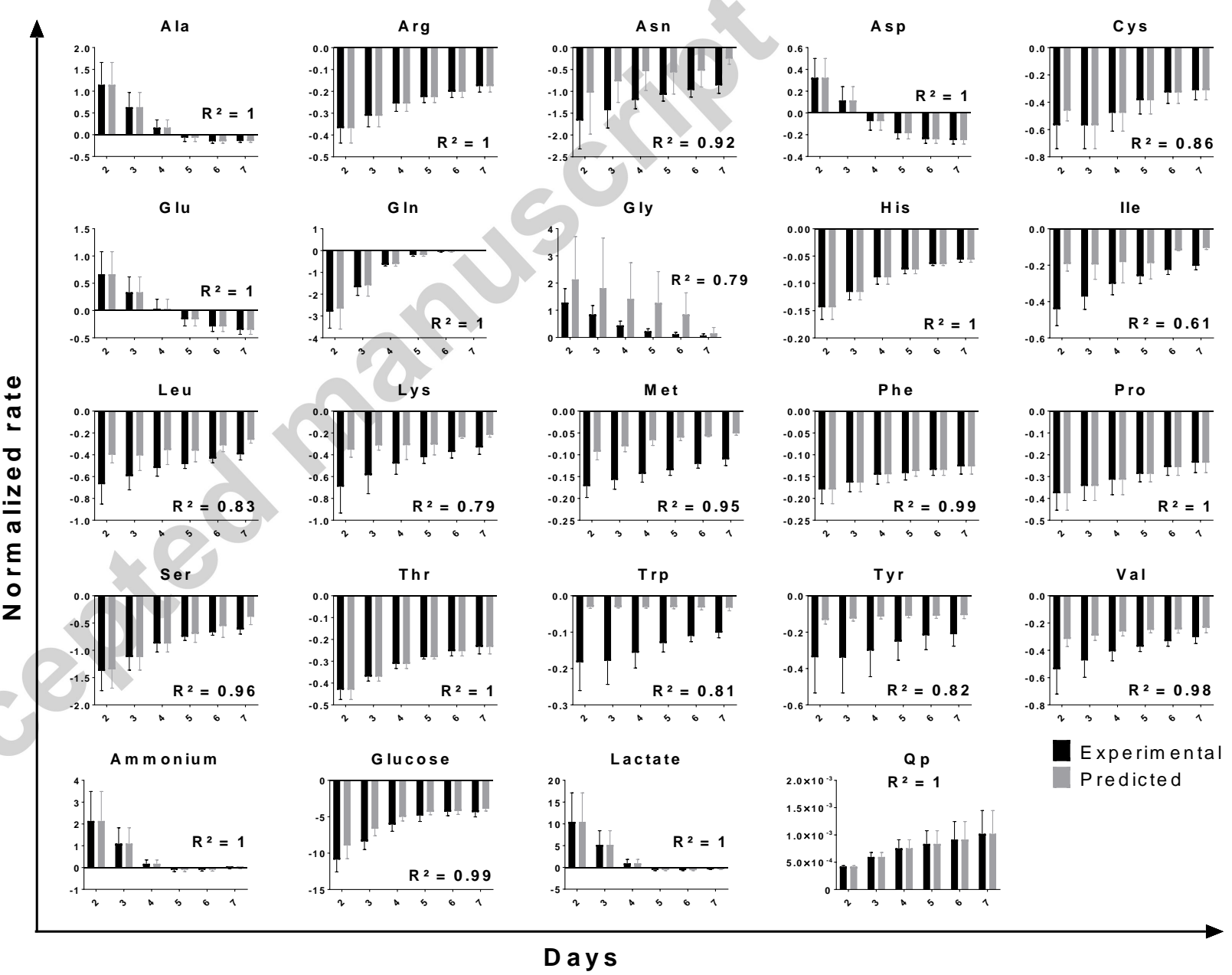

Fig. 3. (A) Correlation coefficient $\left(r^{2}\right)$ of predicted growth and specific productivity $(Q p)$ with experimental data. Growth rate was predicted from day 2 to 7 and $Q p$ from day 6 to 9, using respectively maximization of biomass production and antibody production as the objective function. Average value of the 4 independent predictions was used to determine squared correlation coefficient. (B) Comparison between experimental fluxes and predicted fluxes in case the objective function chosen is maximization of biomass. Normalized consumption (negative) and production (positive) rates of extracellular metabolites at each day of the cell culture. Coefficient of determination ( $r$-squared) was calculated from the regression line of the average experimental and the average predicted values. Error bars denote standard deviation of the mean $(n=4)$. Information about the method applied for modeling is detailed in section 2.2 . 
3.2.2.2. Analysis of fluxes that drive metabolic switch from production to net consumption gives better understanding for medium optimization

Metabolites such as alanine, aspartate, lactate and glutamate are produced in the early stage of the culture and a switch from production to consumption is observed at day 4 (Fig. 3). The accumulation of some of the produced metabolites are responsible for a decreased cell growth and antibody productivity (Duarte et al., 2014; Lao and Toth, 1997; Ozturk et al., 1992). As the mechanisms that trigger this metabolic shift are unknown (Hartley et al., 2018), the model was used to analyze the rewiring of metabolic pathway and what can be done at the level of medium composition to avoid any negative impact on cell growth.

\subsection{Flux predictions describe alanine and lactate switch from production to consumption throughout the culture}

Based on the predictions, alanine is entirely produced by alanine transaminase from day 2 to 5 and imported from the medium towards the end of the culture. The shift from production to consumption at day 4 can be correlated to lactate shift, as lactate is no longer available for conversion to pyruvate, cell metabolism redirects pyruvate fluxes to maintain flow to mitochondrial citric acid cycle. In silico, alanine is consumed to form proteins for biomass formation as well as the low and heavy chain of the antibody, thus predicted alanine metabolism is in agreement with literature (Suppl. Fig. 6) (Pereira et al., 2018; Sellick et al., 2015). As pyruvate resulting from intense early glycolytic activity is converted to both lactate and alanine, pyruvate and glucose concentration in culture medium should be controlled at a low level at the beginning of the process.

3.2.2.2.2.

Genome scale model highlights important role of asparaginase in ammonium production

Accumulation of ammonium in the production medium has been shown to be inhibitory for cell growth and antibody productivity (Chen and Harcum, 2005; McQueen and Bailey, 1990; Yang and Butler, 2000), thus the model was used to identify the different source of ammonium production during the process. From day 2 to 5 , ammonium is mainly produced by glutaminase, which catalyzes the hydrolysis of glutamine to glutamate and ammonia (Suppl. Fig. 7). This initially high secretion of ammonium adds up to an overall constant ammonium production from 
asparaginase and cysteine desulfhydrase. Ammonium is highly secreted at the early exponential phase, and a switch is observed after exhaustion of glutamine in the medium. As glutamine requirement increases, glutamine synthetase is responsible for the minor NH4 uptake observed day 5 , 6 and 7.

What is interesting to notice in these predictions, is the relatively high and steady production of ammonium due to asparaginase activity. This observation seems to be confirmed by different studies (Pereira et al., 2018; Selvarasu et al., 2012). In order to assess the impact of ammonium production from asparagine, we performed an experiment with higher amount of asparagine in the production medium and/or in the feed. As shown in Suppl. Fig. 8, higher asparagine concentration in the medium leads to a consistently higher ammonium production rate towards the end of the process. This elevated ammonium release is more likely to be the product degradation of asparaginase, as no ammonium accumulation was measured in the medium alone supplemented with asparagine, kept under similar culture condition, thus excluding a natural degradation of asparagine in the medium (data not shown). A possible way to reduce ammonium secretion would be to decrease asparagine concentration, or to increase the concentration of the product of asparaginase, aspartate, to avoid generation of additional by-product in the medium.

\subsection{Extracellular glutamine depletion triggers redirection of fluxes to maintain glutamate requirements}

Predicted glutamate catabolism is represented in Suppl. Fig. 9. Reactions catabolizing glutamate to replenish TCA cycle were grouped together (phosphoserine transaminase, glutamate dehydrogenase and aspartate transaminase). The predicted consumption rate of glutamate for global replenishment TCA cycle intermediates is higher than for protein synthesis at all days.

As glutamate is required for synthesis of glutamine from day 5, glutamine synthetase flux is activated to synthesize glutamine for glutamate and thus its biosynthetic requirement increases to maintain elevated TCA activity and protein synthesis rate. Experimental glutamate uptake rate increases from day 5 until day 7 to maintain energy and growth requirements. In the model glutamate contribution for protein synthesis remains the same (about $10 \%$ of the net consuming fluxes). 


\section{ACCEPTED MANUSCRIPT}

Among the amino acids imported from the medium, arginine, phenylalanine, proline, tryptophan, tyrosine, and valine are predicted to be entirely utilized for synthesis of biomass and antibody from day 2 to 7 . However at day 5 and 6 , isoleucine, leucine, lysine and proline are catabolized to produce glutamate respectively by isoleucine transaminase, leucine transaminase and saccharopine dehydrogenase. The predictions indicate that these metabolites are partially compensating increased glutamate requirement from day 5.

\subsubsection{Conclusion of the analysis of predicted phenotype and exometabolomics rates}

Amino acid metabolism consists of a complex network of reactions, and their catabolism during cell culture can vary according to the environmental conditions. The predictions obtained for this process regarding catabolism of $96 \%$ of the amino acids are in correlation with the general use of the nutrients in $\mathrm{CHO}$ cell culture (Grohmann and Bronte, 2010). As demonstrated, genome scale models are the most suitable tool to analyze nutrient catabolism and sub consequent activation of reactions responsible for higher by-product secretions.

As a conclusion to this assessment study of the tailored genome scale model, the improvements and manual modifications performed have led to a functional model that can be successfully applied to predict metabolic capabilities of industrial CHO cell producers in a robust manner. Both the curated model and the methodology for simulation of the environmental conditions are validated, and further analysis is performed to gain additional biological insights.

\subsubsection{In silico analysis of intracellular flux distribution}

The activity of 18 reactions covering important metabolic branch points are shown in Fig. 4. 


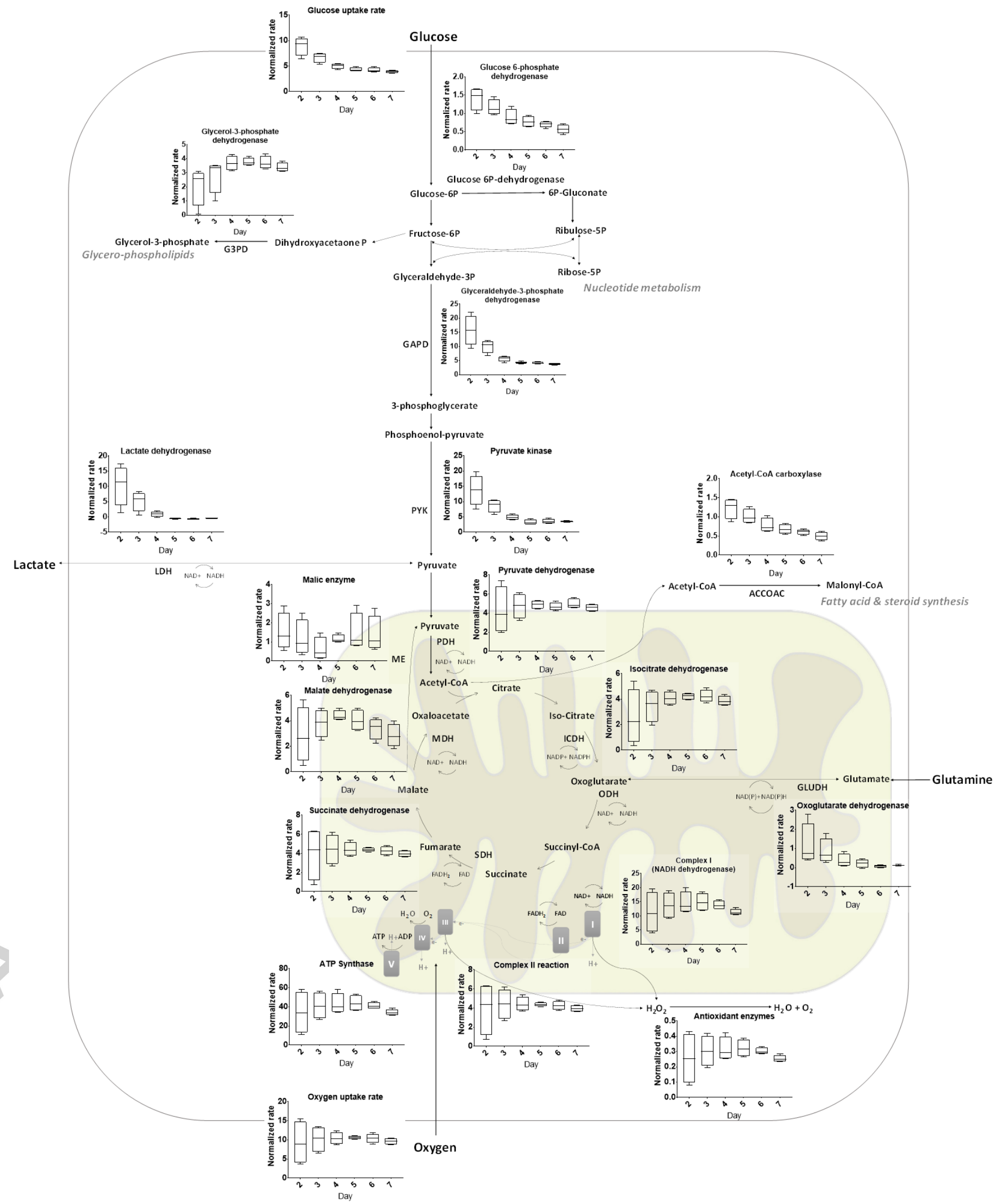

Fig. 4. Major pathways of central carbon metabolism and predicted distribution of normalized intracellular fluxes from day 2 to 7 for independent $2 \mathrm{~L}$ bioproduction processes ( $n=4$ biological replicates). 
A first metabolic phase can be identified from day 2 to 4 , characterized by a high glycolytic and glutaminolytic metabolism. On day 2 and 3 respectively, $76 \%$ and $60 \%$ of pyruvate produced via glycolysis is secreted as lactate, which generates 2 moles of ATP per mole of glucose consumed instead of 36 through oxidative phosphorylation. Although the energetic yield is lower compared to oxidative phosphorylation, the high flux through aerobic glycolysis can produce ATP in large quantities and at a fast rate, as NAD+ recovered from lactate dehydrogenase can be used to maintain glycolysis. On the other hand, glutamine is largely used to fuel the TCA cycle with 2-oxoglutarate, which complete oxidation contributes to ATP production by the TCA cycle and electron transport chain.

High predicted flux through pentose phosphate pathway (PPP) in the first days allows generation of key intermediates for nucleotide metabolism (ribose-5-phosphate), for fatty acid synthesis and for reduction of oxidative stress with reducing NADPH, which provides the cell with bioenergetic resources that are essential for proliferation. PPP flux, characterized by a linear decrease towards the stationary phase, can be compared with experimental results obtained with a 13C-based MFA study conducted on a fed batch cell culture process with CHO-K1 cells (Ahn and Antoniewicz, 2011). By day 6, a significant decrease of NADH and NADPH production at different branches of the metabolism can be linked to a drop of activity for complex I and antioxidant enzymes. The decreased enzymatic activity of the following reactions could worsen the metabolic burden towards the end of cell proliferation: glucose 6-phosphate dehydrogenase, malate dehydrogenase, complex I of oxidative phosphorylation and activity of antioxidant enzymes.

A second phase can be observed after day 4 , with the exhaustion of glutamine possibly linked to an increased activity of IDH in order to compensate a decreasing supply of oxoglutarate, and to a decreased activity of malate dehydrogenase, as pyruvate supply from glycolysis decreases and its consumption rate through TCA cycle remains constant. Supplementing glutamine in the medium would not be an interesting strategy as glutamine metabolism releases ammonia, which accumulation in the medium has been reported to be inhibitory or toxic to cells. A different feeding strategy could be considered here with controlled additions of glutamine to maintain it at a low concentration from day 4.

Flow through glycolysis decreases with decreased growth rate towards day 7, similarly to the oxidative branch of the PPP and to acetyl-CoA carboxylase, thus associated to a decreased production of fatty acids synthesis required for cell growth. However flow through TCA cycle is more or less constant, except for malic enzyme and malate dehydrogenase which are balancing each other and are compensating a decreased income of pyruvate through glycolysis (pyruvate kinase) from day 4, by re-directing malate to synthesis of pyruvate instead of oxaloacetate. Although 
predicted oxygen uptake rate remains almost constant with a low variation between the run, ATP synthase activity significantly decreases in the

last days following the same trend as the complex I. According to these predictions, cell death seems to be preceded by an overall lower rate of NADH production, possibly a triggering signal for apoptosis.

\section{Conclusion}

The initial CHO cell line specific GSM was modified in order to cope with constraints that are either not available or not applied in the chosen mathematical representation of the metabolic network. The improved model was also modified by considering specific metabolic traits observed in high producing cell lines. Thus this network was carefully and manually curated, firstly by identifying reactions that could not be modeled accurately and by determining a rationale for simplification, and secondly by incorporating in the model information specific to industrial cells used for recombinant protein production, regarding the adaptation of cells to oxidative stress under elevated oxygen conditions and lipid storage. The results presented here showed that the flux distributions of the curated CHO DG44 specific line GSM was biologically meaningful and yielded insights into cell metabolism. Predicted growth rate shows a very good correlation coefficient of 0.91 with experimental growth, and $96 \%$ of the extracellular metabolites measured showed a coefficient higher than 0.8 . Based on this high correlation the analysis of the metabolic network was considered valid from day 2 to 7 , and the predictions allowed to identify potential ways for medium optimization, such as reducing the amount of asparagine. With the current knowledge integrated in the metabolic network, observations that can be drawn from in silico analysis of cell metabolism at different days for the 4 independent $2 \mathrm{~L}$ batches open up the opportunity for biological discovery and understanding of cell machinery. During cell culture process, amino acid required for protein and biomass synthesis are predicted to be increasingly redirected in anaplerotic reactions to support energy requirements. Intracellular analysis of cell metabolism can help to identify bottlenecks and breaking point preceding death phase. The predicted evolution of energy metabolism is highly impacted by the activity of pathways that produce and consume NAD+ and by the maintenance of a cellular NAD+/NADH balance. This ratio could be changed by overexpressing specific enzymes to augment internal cellular energy metabolism towards the end of the exponential phase, as it has already been 
performed with $\mathrm{CHO}$ cells (Gupta et al., 2017). If successful, we can imagine achieving extended culture time and delaying cell death, which

feeds high expectations for an increased cell productivity (Lee et al., 1998).

\section{Funding}

This work was supported by an H2020 Marie Sklodowska-Curie Actions ITN, grant number 642663.

\section{References}

2002. Molecular Biology of the Cell (4th Ed). Journal of Biological Education. 37, 45-47.

Ahn, W. S., Antoniewicz, M. R., 2011. Metabolic flux analysis of CHO cells at growth and non-growth phases using isotopic tracers and mass spectrometry. Metab Eng. 13, 598-609.

Ali, A. S., Raju, R., Ray, S., Kshirsagar, R., Gilbert, A., Zang, L., Karger, B. L., 2018. Lipidomics of CHO Cell Bioprocessing: Relation to Cell Growth and Specific Productivity of a Monoclonal Antibody. Biotechnol J. e1700745.

Becker, S. A., Palsson, B. O., 2008. Context-specific metabolic networks are consistent with experiments. PLoS Comput Biol. 4 , e1000082.

Blacker, T. S., Duchen, M. R., 2016. Investigating mitochondrial redox state using NADH and NADPH autofluorescence. Free Radic Biol Med. $100,53-65$.

Bonarius, H. P. J., Hatzimanikatis, V., Meesters, K. P. H., de Gooijer, C. D., Schmid, G., Tramper, J., 1996. Metabolic flux analysis of hybridoma cells in different culture media using mass balances. Biotechnology and Bioengineering. 50, 299-318.

Bowes, T., Singh, B., Gupta, R. S., 2007. Subcellular localization of fumarase in mammalian cells and tissues. Histochem Cell Biol. $127,335-46$.

Boyle, J., 2005. Lehninger principles of biochemistry (4th ed.): Nelson, D., and Cox, M. Biochemistry and Molecular Biology Education. 33 , 74-75.

Burgess, D. J., 2011. Metabolism: Choose your carbon source. Nature Reviews Cancer. 11, 80-81.

Calmels, C., Chintan, J., Nathan, E. L., Malphettes, Andersen, M. R., 2018. Adaption of Generic Metabolic Models to Specific Cell Lines for Improved Modeling of Biopharmaceutical Production and Prediction of Processes. Advanced Biotechnology Book Series. (accepted)

Cacciatore, J. J., Chasin, L. A., Leonard, E. F., 2010. Gene amplification and vector engineering to achieve rapid and high-level therapeutic protein production using the Dhfr-based CHO cell selection system. Biotechnol Adv. 28, 673-81.

Cecchini, G., 2003. Function and structure of complex II of the respiratory chain. Annu Rev Biochem. 72, 77-109.

Chen, P., Harcum, S. W., 2005. Effects of amino acid additions on ammonium stressed CHO cells. J Biotechnol. 117, $277-86$.

De Jesus, M., Wurm, F. M., 2011. Manufacturing recombinant proteins in kg-ton quantities using animal cells in bioreactors. Eur J Pharm Biopharm. 78, 184-8.

Deberardinis, R. J., Sayed, N., Ditsworth, D., Thompson, C. B., 2008. Brick by brick: metabolism and tumor cell growth. Curr Opin Genet Dev. 18 , 54-61.

Deshpande, R. R., Heinzle, E., 2004. On-line oxygen uptake rate and culture viability measurement of animal cell culture using microplates with integrated oxygen sensors. Biotechnology Letters. 26, 763-767.

Duarte, N. C., Becker, S. A., Jamshidi, N., Thiele, I., Mo, M. L., Vo, T. D., Srivas, R., Palsson, B. O., 2007. Global reconstruction of the human metabolic network based on genomic and bibliomic data. Proc Natl Acad Sci U S A. 104, 1777-82.

Duarte, T. M., Carinhas, N., Barreiro, L. C., Carrondo, M. J., Alves, P. M., Teixeira, A. P., 2014. Metabolic responses of CHO cells to limitation of key amino acids. Biotechnol Bioeng. 111, 2095-106.

Ebrahim, A., Lerman, J. A., Palsson, B. O., Hyduke, D. R., 2013. COBRApy: COnstraints-Based Reconstruction and Analysis for Python. BMC Syst Biol. 7, 74.

Farese, R. V., Jr., Walther, T. C., 2009. Lipid droplets finally get a little R-E-S-P-E-C-T. Cell. 139, 855-60.

Geoghegan, D., Arnall, C., Hatton, D., Noble-Longster, J., Sellick, C., Senussi, T., James, D. C., 2018. Control of Amino Acid Transport into CHO Cells. Biotechnol Bioeng.

GM., C., 2000. Transport of Small Molecules. . The Cell: A Molecular Approach. 2nd edition. . Sunderland (MA): Sinauer Associates.

Grohmann, U., Bronte, V., 2010. Control of immune response by amino acid metabolism. Immunol Rev. 236, $243-64$.

Gupta, S. K., Srivastava, S. K., Sharma, A., Nalage, V. H. H., Salvi, D., Kushwaha, H., Chitnis, N. B., Shukla, P., 2017. Metabolic engineering of CHO cells for the development of a robust protein production platform. PLoS One. 12, e0181455.

Ha, T. K., Hansen, A. H., Kol, S., Kildegaard, H. F., Lee, G. M., 2018. Baicalein Reduces Oxidative Stress in CHO Cell Cultures and Improves Recombinant Antibody Productivity. Biotechnol J. 13, e1700425. 


\section{ACCEPTED MANUSCRIPT}

Hansen, H. A., Emborg, C., 1994. Extra- and intracellular amino acid concentrations in continuous Chinese hamster ovary cell culture. Appl Microbiol Biotechnol. 41, 560-4.

Hartley, F., Walker, T., Chung, V., Morten, K., 2018. Mechanisms driving the lactate switch in Chinese hamster ovary cells. Biotechnol Bioeng.

Hastie, T., Tibshirani, R., Friedman, J., 2009. The Elements of Statistical Learning. Springer-Verlag New York.

Hefzi, H., Ang, K. S., Hanscho, M., Bordbar, A., Ruckerbauer, D., Lakshmanan, M., Orellana, C. A., Baycin-Hizal, D., Huang, Y., Ley, D., Martinez, V. S., Kyriakopoulos, S., Jimenez, N. E., Zielinski, D. C., Quek, L. E., Wulff, T., Arnsdorf, J., Li, S., Lee, J. S., Paglia, G., Loira, N., Spahn, P. N., Pedersen, L. E., Gutierrez, J. M., King, Z. A., Lund, A. M., Nagarajan, H., Thomas, A., Abdel-Haleem, A. M., Zanghellini, J., Kildegaard, H. F., Voldborg, B. G., Gerdtzen, Z. P., Betenbaugh, M. J., Palsson, B. O., Andersen, M. R., Nielsen, L. K., Borth, N., Lee, D. Y., Lewis, N. E., 2016. A Consensus Genomescale Reconstruction of Chinese Hamster Ovary Cell Metabolism. Cell Syst. 3, 434-443 e8.

Ho, Y. S., Xiong, Y., Ma, W., Spector, A., Ho, D. S., 2004. Mice lacking catalase develop normally but show differential sensitivity to oxidant tissue injury. J Biol Chem. 279, 32804-12.

Jefferis, R., 2016. Posttranslational Modifications and the Immunogenicity of Biotherapeutics. J Immunol Res. 2016, 5358272.

Kaas, C. S., Fan, Y., Weilguny, D., Kristensen, C., Kildegaard, H. F., Andersen, M. R., 2014. Toward genome-scale models of the Chinese hamster ovary cells: incentives, status and perspectives. Pharmaceutical Bioprocessing. 2, 437-448.

Kanehisa, M., Furumichi, M., Tanabe, M., Sato, Y., Morishima, K., 2017. KEGG: new perspectives on genomes, pathways, diseases and drugs. Nucleic Acids Res. 45, D353-D361.

Kanehisa, M., Goto, S., 2000. KEGG: kyoto encyclopedia of genes and genomes. Nucleic Acids Res. 28, 27-30.

Kanehisa, M., Sato, Y., Kawashima, M., Furumichi, M., Tanabe, M., 2016. KEGG as a reference resource for gene and protein annotation. Nucleic Acids Res. 44, D457-62.

Keizer, H. G., van Rijn, J., Pinedo, H. M., Joenje, H., 1988. Effect of endogenous glutathione, superoxide dismutases, catalase, and glutathione peroxidase on adriamycin tolerance of Chinese hamster ovary cells. Cancer Res. 48, 4493-7.

Khetan, A., Huang, Y. M., Dolnikova, J., Pederson, N. E., Wen, D., Yusuf-Makagiansar, H., Chen, P., Ryll, T., 2010. Control of misincorporation of serine for asparagine during antibody production using $\mathrm{CHO}$ cells. Biotechnol Bioeng. 107, 116-23.

Lao, M. S., Toth, D., 1997. Effects of ammonium and lactate on growth and metabolism of a recombinant Chinese hamster ovary cell culture. Biotechnol Prog. 13, 688-91.

Le Fourn, V., Girod, P. A., Buceta, M., Regamey, A., Mermod, N., 2014. CHO cell engineering to prevent polypeptide aggregation and improve therapeutic protein secretion. Metab Eng. 21, 91-102.

Lee, Y. S., Nakajima, H., Chang, Y. C., Park, K. I., Mitsui, Y., Magae, J., Saida, K., 1998. Alleviation of apoptosis by serum in Chinese hamster ovary cells ectopically expressing human Fas antigen. Mol Cells. 8, 272-9.

Lenaz, G., Genova, M. L., 2010. Structure and organization of mitochondrial respiratory complexes: a new understanding of an old subject. Antioxid Redox Signal. 12, 961-1008.

Lewis, N. E., Hixson, K. K., Conrad, T. M., Lerman, J. A., Charusanti, P., Polpitiya, A. D., Adkins, J. N., Schramm, G., Purvine, S. O., Lopez-Ferrer, D., Weitz, K. K., Eils, R., Konig, R., Smith, R. D., Palsson, B. O., 2010. Omic data from evolved E. coli are consistent with computed optimal growth from genomescale models. Mol Syst Biol. 6, 390.

Li, F., Vijayasankaran, N., Shen, A., Kiss, R., Amanullah, A., 2014. Cell culture processes for monoclonal antibody production. mAbs. 2, $466-479$.

Machado, D., Herrgard, M., 2014. Systematic evaluation of methods for integration of transcriptomic data into constraint-based models of metabolism. PLoS Comput Biol. 10, e1003580.

Matyash, V., Liebisch, G., Kurzchalia, T. V., Shevchenko, A., Schwudke, D., 2008. Lipid extraction by methyl-tert-butyl ether for high-throughput lipidomics. J Lipid Res. 49, 1137-46.

McQueen, A., Bailey, J. E., 1990. Effect of ammonium ion and extracellular $\mathrm{pH}$ on hybridoma cell metabolism and antibody production. Biotechnol Bioeng. 35, 1067-77.

Opdam, S., Richelle, A., Kellman, B., Li, S., Zielinski, D. C., Lewis, N. E., 2017. A Systematic Evaluation of Methods for Tailoring Genome-Scale Metabolic Models. Cell Syst. 4, 318-329 e6.

Ozturk, S. S., Riley, M. R., Palsson, B. O., 1992. Effects of ammonia and lactate on hybridoma growth, metabolism, and antibody production. Biotechnol Bioeng. 39, 418-31.

Pan, X., Dalm, C., Wijffels, R. H., Martens, D. E., 2017. Metabolic characterization of a CHO cell size increase phase in fed-batch cultures. Appl Microbiol Biotechnol. 101, 8101-8113.

Pereira, S., Kildegaard, H. F., Andersen, M. R., 2018. Impact of CHO Metabolism on Cell Growth and Protein Production: An Overview of Toxic and Inhibiting Metabolites and Nutrients. Biotechnol J.

Pieper, L. A., Strotbek, M., Wenger, T., Gamer, M., Olayioye, M. A., Hausser, A., 2017. Secretory pathway optimization of CHO producer cells by coengineering of the mitosRNA-1978 target genes CerS2 and Tbc1D20. Metab Eng. 40, 69-79. 
Placzek, S., Schomburg, I., Chang, A., Jeske, L., Ulbrich, M., Tillack, J., Schomburg, D., 2017. BRENDA in 2017: new perspectives and new tools in BRENDA. Nucleic Acids Research. 45, D380-D388.

Quek, L. E., Dietmair, S., Hanscho, M., Martinez, V. S., Borth, N., Nielsen, L. K., 2014. Reducing Recon 2 for steady-state flux analysis of HEK cell culture. J Biotechnol. 184, 172-8.

Rahimpour, A., Vaziri, B., Moazzami, R., Nematollahi, L., Barkhordari, F., Kokabee, L., Adeli, A., Mahboudi, F., 2013. Engineering the cellular protein secretory pathway for enhancement of recombinant tissue plasminogen activator expression in Chinese hamster ovary cells: effects of CERT and XBP1s genes. J Microbiol Biotechnol. 23, 1116-22.

Reinhart, D., Damjanovic, L., Kaisermayer, C., Kunert, R., 2015. Benchmarking of commercially available CHO cell culture media for antibody production. Appl Microbiol Biotechnol. 99, 4645-57.

Segre, D., Vitkup, D., Church, G. M., 2002. Analysis of optimality in natural and perturbed metabolic networks. Proc Natl Acad Sci U S A. 99, $15112-7$.

Sellick, C. A., Croxford, A. S., Maqsood, A. R., Stephens, G. M., Westerhoff, H. V., Goodacre, R., Dickson, A. J., 2015. Metabolite profiling of CHO cells: Molecular reflections of bioprocessing effectiveness. Biotechnol J. 10, 1434-45.

Selvarasu, S., Ho, Y. S., Chong, W. P., Wong, N. S., Yusufi, F. N., Lee, Y. Y., Yap, M. G., Lee, D. Y., 2012. Combined in silico modeling and metabolomics analysis to characterize fed-batch CHO cell culture. Biotechnol Bioeng. 109, 1415-29.

Templeton, N., Dean, J., Reddy, P., Young, J. D., 2013. Peak antibody production is associated with increased oxidative metabolism in an industrially relevant fed-batch $\mathrm{CHO}$ cell culture. Biotechnol Bioeng. 110, 2013-24.

Thiele, I., Palsson, B. O., 2010. A protocol for generating a high-quality genome-scale metabolic reconstruction. Nat Protoc. 5, 93-121.

Toroghi, M. K., Cluett, W. R., Mahadevan, R., 2016. A Multi-Scale Model of the Whole Human Body based on Dynamic Parsimonious Flux Balance Analysis. IFAC-PapersOnLine. 49, 937-942.

Turrens, J. F., 2003. Mitochondrial formation of reactive oxygen species. J Physiol. 552, 335-44.

Vriezen, N., 1998. Physiology of mammalian cells in suspension culture. Doctoral thesis.

Wen, D., Vecchi, M. M., Gu, S., Su, L., Dolnikova, J., Huang, Y. M., Foley, S. F., Garber, E., Pederson, N., Meier, W., 2009. Discovery and investigation of misincorporation of serine at asparagine positions in recombinant proteins expressed in Chinese hamster ovary cells. J Biol Chem. 284, $32686-94$.

Weydert, C. J., Cullen, J. J., 2010. Measurement of superoxide dismutase, catalase and glutathione peroxidase in cultured cells and tissue. Nat Protoc. 5, 51-66.

Xie, L., Wang, D. I., 1994. Applications of improved stoichiometric model in medium design and fed-batch cultivation of animal cells in bioreactor. Cytotechnology. 15, 17-29.

Yang, M., Butler, M., 2000. Effect of ammonia on the glycosylation of human recombinant erythropoietin in culture. Biotechnol Prog. 16, 751-9.

Zomorrodi, A. R., Suthers, P. F., Ranganathan, S., Maranas, C. D., 2012. Mathematical optimization applications in metabolic networks. Metab Eng. 14, 672-86.

Zupke, C., Stephanopoulos, G., 1995. Intracellular flux analysis in hybridomas using mass balances and in vitro (13)C nmr. Biotechnol Bioeng. 45, $292-303$.

Highlights

- A genome scale model specific for CHO DG44 cell line was manually adapted to the metabolism of industrial cell lines used for the production of recombinant therapeutic proteins

- A modeling approach was defined for prediction of intracellular fluxes at different days of an industrial cell culture process

- The curated model can be used to get deeper insight into metabolism of cells cultivated in industrial processes 